DEPARTMENT OF THE INTERIOR

U.S. GEOLOGICAL SURVEY

Vitrinite Reflectance Values of Coal from Drill-Hole Cuttings from the Fruitland and Menefee Formations,

San Juan Basin, New Mexico

by

James E. Fassett ${ }^{1}$ and Vito F. Nuccio ${ }^{1}$

Open-File Report 90-290

This report is preliminary and has not been reviewed for conformity with U.S. Geological survey editorial standards (or with the North American stratigraphic Code). Any use of trade, product, or firm names is for descriptive purposes only and does not imply endorsement by the U.S. Government.

${ }^{1}$ U.S. Geological Survey, P.O. Box 25046, MS 939, DFC Denver, Colorado 80225 


\title{
Vitrinite Reflectance Values of Coal from Drill-Hole Cuttings from the Fruitland and Menefee Formations, \\ San Juan Basin, New Mexico
}

\author{
James E. Fassett and Vito F. Nuccio
}

\section{INTRODUCTION}

In May 1977, the discovery well for the cedar Hill Fruitland Basal Coal field was completed. Cedar Hill was the first gas field in the san Juan Basin to produce methane exclusively from Upper Cretaceous Fruitland Formation coal beds. (Earlier wells had produced gas from Fruitland coal beds, but all of those wells were characterized as producing from the Fruitland and Pictured Cliffs sandstone, undivided.) Following the cedar Hill discovery, hundreds of additional coal-bed methane wells have been completed in the Fruitland Formation in the colorado and New Mexico parts of the san Juan Basin. This development has stimulated many new research projects designed to better understand the physical and chemical characteristics of Fruitland coal beds. This report is the result of one such project; a cooperative effort between the New Mexico oil Conservation Division (NMOCD) and the U.S. Geological survey (USGS). In 1988, the NMOCD established requirements that all oil and gas operators drilling oil or gas test holes through coal-bearing strata in the New Mexico part of the san Juan Basin send drill-hole cuttings of coal to the USGS in Denver. These samples are being processed and analyzea for vitrinite reflectance values of the contained coals.

This report lists vitrinite reflectance values (\% $\mathrm{Rm}$ ) for coal cuttings for the first 90 wells processed. Because this is an ongoing project, additional USGS open File reports simiiar to this one will be periodically published. The data are tabulated by township, range, and section for each well. The depths in feet of coal-cuttings samples were provided by the companies drilling the wells and no attempt was made to correct or adjust these depths precisely to specific coal beds on geophysical logs. In working with drill-hole cuttings, the possibility always exists that samples reported to be from a given depth interval have been contaminated by material from a shallower depth interval as the result of caving up-hole. No evaluation regarding such contamination is included in this report. We anticipate that as additional samples are processed and the overall data-set becomes larger, anomalous values resulting from such contamination will stand out and can thus be identified and deleted from the final data set. Therefore, the data in table 1 are preliminary. A forthcoming report will interpret these data and include a discussion of the burtal and thermal history of Upper Cretaceous rocks of the San Juan Basin.

For this study, coal was prepared by crushing the sample, mounting it in epoxy on a microscope slide, planing it off when hardened, and polishing it. For all samples, percent mean random 
vitrinite reflectance (\%Rm) was measured from randomly oriented indigenous vitrinite grains in immersion oil using a non-rotating stage zeiss Universal research microscope. Plane-polarized incident white light and a $546 \mathrm{~nm}$ monochromatic filter were used (for a complete explanation see stach and others, 1982). The number of readings per sample varied depending on the amount of clean, indigenous vitrinite in the sample. In most of the samples vitrinite was abundant and the number of readings per sample was between 30 and 40 . If the number of readings for a sample is greater than 30 and the standard deviation is relatively small, the mean vitrinite reflectance value is statistically valid.

\section{GENERAL GEOLOGY}

The San Juan Basin is in the northwest part of New Mexico and the southwest part of Colorado (fig. 1). As depicted on figure 1 , the basin consists of three areas; the central basin, the Four Corners Platform, and the Chaco slope. The central basin is outlined around its west, north, and east sides by a monoclinal rim or hogback. The southern boundary of the central basin has no structural element marking it and is commonly placed approximately along the outcrop of the upper cretaceous Pictured cliffs sandstone. The central basin has a diameter of about 100 miles $(60 \mathrm{~km})$ and an area of about 7,500 square miles $(19,500$ square $\mathrm{km}$ ).

The basin is asymmetric with its deepest part in the northeast (fig. 2). Structural dips around the northwestern, northern, and eastern edges of the basin are 20-40 degrees, whereas dips on the western and southern sides average less than a degree.

The Upper Cretaceous rocks of the san Juan Basin comprise a sequence of intertonguing, time-transgressive, continental and marine rocks. These rocks were deposited in an embayment of the western shore of the Western Interior Cretaceous seaway during several cycles of shoreline transgression and regression. shorelines trended generally northwest during deposition of upper cretaceous rocks in the San Juan Basin area.

\section{SAN JUAN BASIN COALS}

The following summary of San Juan Basin coals is modified from Fassett (1989).

In the San Juan Basin, most of the Upper Cretaceous continental strata overlying regressive-marine shoreface sandstones contain coal beds in their lower parts. Examples are the Dilco Coal Member of the Crevasse Canyon Formation which overlies the regressive Gallup Sandstone; the Gibson Coal Member of the Crevasse Canyon which overlies the regressive Dalton 


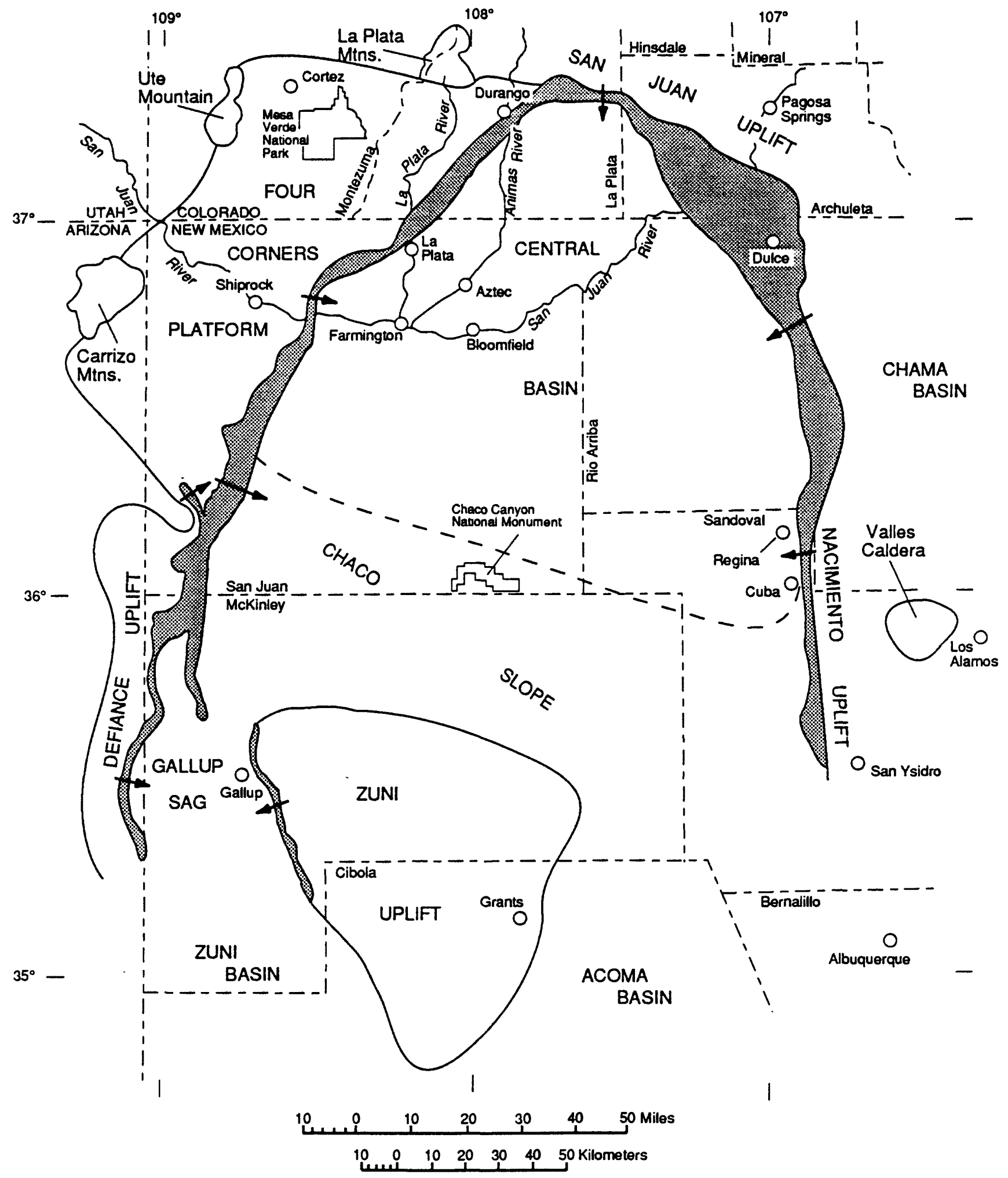

Figure 1--Map showing the structural elements of the san Juan Basin. structural elements were generalized from a more detailed structure map of the basin area by Thaden and zech (1984). The patterned areas are areas of relatively steep dip (monoclines); arrows indicate direction of dip; the dashed line that separates the central Basin from the Chaco slope is located approximately along the outcrop of the Pictured Cliffs Sandstone. 


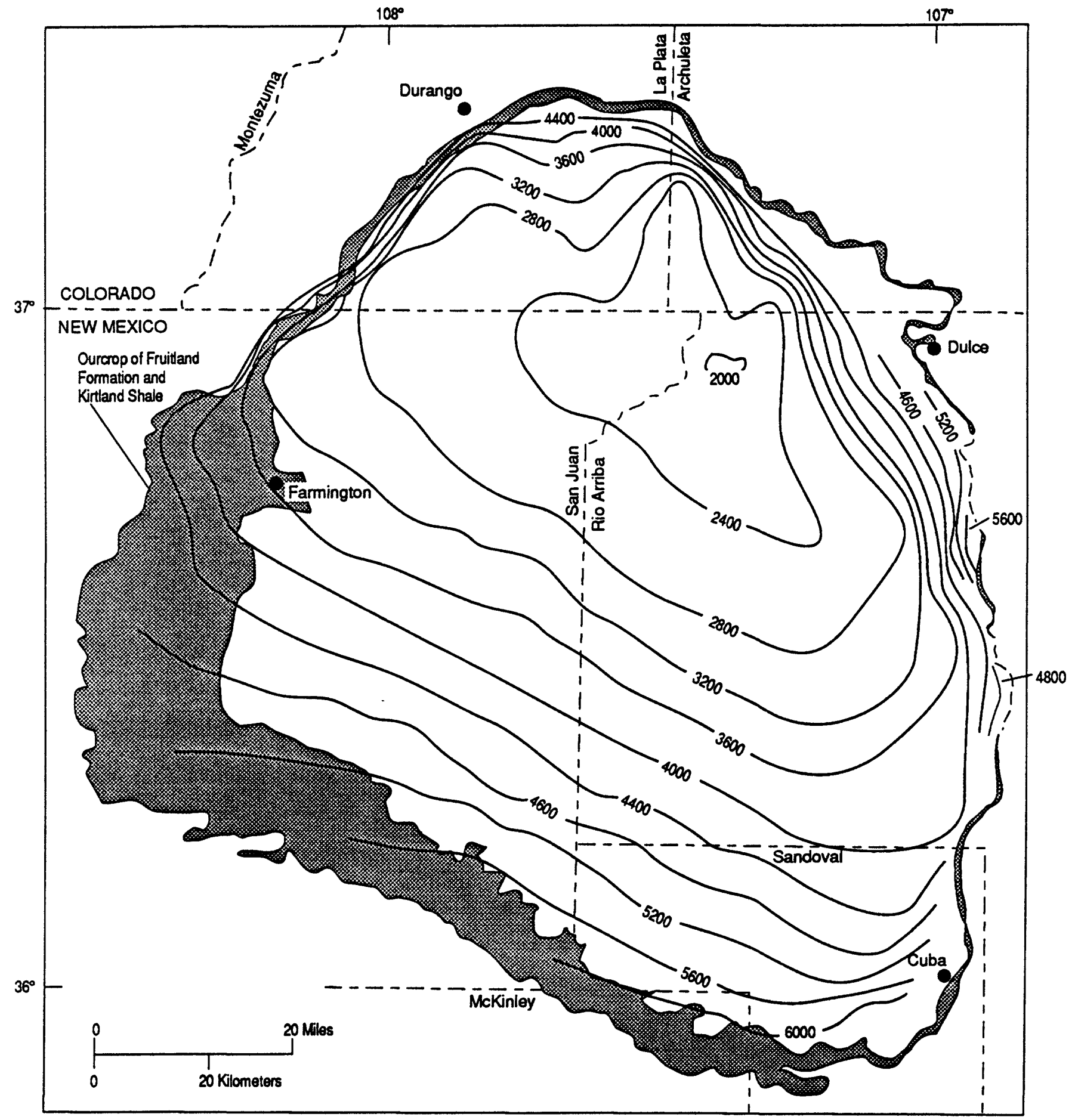

Figure 2--Map showing the outcrop of the Fruitland Formation and Kirtland Shale and the structure of the san Juan Basin. Contours are drawn on the Huerfanito Bentonite Bed of the Lewis Shale; contour interval is 400 feet $(120 \mathrm{~m})$. Datum is mean sea level. From Fassett (1989). 
Sandstone Member of the Crevasse Canyon; the lower part of the Menefee Formation (Cleary Coal Member of the Menefee in the southwest part of the basin) which overlies the regressive point Lookout Sandstone; and the lower part of the Fruitland Formation which overlies the regressive Pictured cliffs sandstone. Some thin, discontinuous coal beds are associated with the Dakota sandstone and the cliff House sandstone, both of which were deposited during generally transgressive events. An important coal-bearing unit in the upper part of the Menefee Formation intertongues with the La Ventana Tongue of the cliff House Sandstone. (This unit is called the "Hogback Mountain Tongue" in some reports, for example, whyte and Shomaker, 1977.) The coal beds in this unit were formed in association with a regressivemarine sandstone near the base of the La Ventana (Fassett, 1977, fig. 2).

The major coal-bearing unit in the san Juan Basin is the Fruitland Formation. The Fruitland contains more than 200 billion tons of coal. Fruitland coals crop out around the rim of the basin, except for local areas along the east-central rim. In a small area in the north-central part of the basin, these coals are at depths of more than 4,000 feet $(1,220 \mathrm{~m})$.

The Menefee Formation is the second largest coal-bearing rock unit in the basin; it is present over a much larger part of the basin than is the Fruitland Formation. The Menefee, the middle unit of the Mesaverde Group, underlies all of the central basin and crops out around the monoclinal rim of the central basin around its western, northern, and eastern parts. It also crops out over a large part of the chaco slope (fig. 1). The first estimate of Menefee coal resources in the san Juan Basin was 12 billion tons (Whyte and shomaker, 1977). This estimate included Menefee coals less than 4,000 feet $(1,220 \mathrm{~m})$ deep in the southern part of the basin. Of this 12 billion tons of Menefee coal, over 11 billion tons were reported to be in the "Hogback Mountain Tongue" of the Menefee Formation. A more recent report (Crist and others, 1989) assessing Menefee coals throughout the entire san Juan Basin, estimates Menefee coal resources to be 145 billion tons, more than a tenfold increase over the earlier estimate. Crist and others (1989) do not explain the discrepancy between their estimate and that of whyte and shoemaker (1977). other studies of the occurrence of Menefee coal in the subsurface of the basin (Fassett, 1986, 1989), have indicated that the Menefee coals are, with the exception of the "Hogback Mountain Tongue", relatively thin and discontinuous, essentially as described by whyte and shoemaker (1977). Fassett (1989) estimated that the total Menefee coal resources might be around 15 billion tons - the 12 billion tons reported by whyte and shoemaker plus another 3 billion tons for the areas not included in the whyte and shoemaker report. 
The Gibson and Dilco Members of the Crevasse Canyon Formation contain the smallest percentage of the basin's commercial coal resources. Gibson coal is present in a relatively small area southwest of the central basin. Dilco coal beds are more extensive, but are relatively thin and discontinuous. Comprehensive resource estimates of Dilco and Gibson coals have not been made, but the total coal tonnage in these units must be relatively small because their coals are generally thin and discontinuous and these units are present in a small part (southern Chaco slope) of the San Juan Basin.

\section{THERMAL MATURITY}

Data on the thermal maturity of the sedimentary rocks in the san Juan Basin was first presented by Fassett and Hinds (1971) and later by Rice (1983). The paper by Fassett and Hinds (1971) presented data on the distribution of the heating values (Btu) of Fruitland coals and on the distribution of the fixed carbon and volatile content of Fruitland Formation coal beds at 65 localities throughout the San Juan Basin. Rice (1983) presented the distribution of vitrinite reflectance values for 19 Fruitland Formation coal samples. These two data sets are in good agreement and indicate that for Fruitland coals 1) volatiles range from around 50 percent to less than 30 percent, 2) moisture- and ash-free heating values range from around 12,000 Btu to nearly 16,000 Btu, 3) coal rank ranges from subbituminous $B$ to medium-volatile bituminous, and 4) vitrinite reflectance values range from less than $0.5 \% \mathrm{Rm}$ to $1.45 \% \mathrm{Rm}$. Meissner, 1984; Bond, 1984; Rice and others, 1988; and Clarkson and Reiter, 1988) subsequently used these same data to evaluate the basin's burial history, heat-flow history, and methane-generation and migration history. A study by Law (written commun., 1990) of the thermal maturity of Upper Cretaceous rocks in the san Juan Basin contains 90 new vitrinite reflectance values for coals and black shales.

These thermal-maturity studies all conclude that thermal maturity increases toward the structural axis of the basin, but that the area of maximum maturity is slightly offset north of the basin axis. Some authors have attributed this offset to conductance of heat to the northern san Juan Basin from a heat source north of the basin in the San Juan volcanic center. Clarkson and Reiter (1988), however, have questioned this concept because of the low thermal conductivity of rock. They have suggested that heat has been brought to the northern part of the basin by hot fluids moving south from the san Juan volcanic center. Law (written commun. 1990) has suggested that deeplyburied heat sources directly beneath the area north of the basin axis are responsible. A simpler solution may be that the greatest depth of burial during the time between the oligocene and the present was slightly north of the present axis of the basin. 


\section{REFERENCES CITED}

Bond, W.A., 1984, Application of Lopatin's method to determine burial history, evolution of the geothermal gradient, and timing of hydrocarbon generation in Cretaceous source rocks in the San Juan Basin, northwestern New Mexico and southwestern Colorado, in Woodward, J., Meissner, F.J., and Clayton, J.L., eds., Hydrocarbon source rocks of the greater Rocky Mountain region: Denver, Colorado, Rocky Mountain Association of Geologists Guidebook, p. 433-447.

Clarkson, G., and Reiter, M., 1988, An overview of geothermal studies in the san Juan Basin, New Mexico and Colorado, in Fassett, J.E., ed., Geology and coal-bed methane resources of the northern San Juan Basin, Colorado and New Mexico: Rocky Mountain Association of Geologists Guidebook, p. 285291.

Crist, T.E., Boyer, C.M., and Kelso, B.S., 1989, A geologic and coalbed methane resource analysis of the Menefee formation in the San Juan Basin, southwestern Colorado and northwestern new Mexico: Society of Petroleum Engineers SPE 18945 , p. 153-160.

Fassett, J.E., 1977, Geology of the Point Lookout, Cliff House, and Pictured Cliffs sandstones of the san Juan Basin, New Mexico and Colorado, in Fassett, J.E., ed., San Juan Basin III: New Mexico Geological society 28 th Field Conference Guidebook, p. 193-197.

1986, The non-transferability of a cretaceous coal model in the San Juan Basin of New Mexico and Colorado, in Lyons, P.C. and Rice, C.I., eds., Paleoenvironmental and tectonic controls in coal-forming basins in the United states: Geological society of America special paper 210, p. 155-171.

1989, Coal-bed methane -- A contumacious, free spirited bride; the geologic handmaiden of coal beds, in Lorenz, J.C., and Lucas, S.G., eds., Energy Frontiers in the Rockies: Albuquerque, New Mexico, Albuquerque Geological society, p. 131-146.

Fassett, J.E., and Hinds, J.S., 1971, Geology and fuel resources of the Fruitland Formation and Kirtland shale of the san Juan Basin, New Mexico and Colorado: U.S. Geological survey Professional Paper 676, $76 \mathrm{p}$. 
Meissner, F.F., 1984, Cretaceous and lower Tertiary coals as sources for gas accumulations in the Rocky Mountain area, in Woodward, J., Meissner, F.F., and Clayton, J.L., eds., Hydrocarbon source rocks of the greater Rocky Mountain region: Denver, Colorado, Rocky Mountain Association of Geologists Guidebook, p. 401-431.

Rice, D.D., 1983, Relation of natural gas composition to thermal maturity and source rock type in san Juan Basin, northwestern New Mexico and southwestern Colorado: American Association of Petroleum Geologists Bulletin, v. 67, no. 8, p. 1199-1218.

Rice, D.D., Threlkeld, C.N., Vuletich, A.K., and Pawlewicz, M.J., 1988, Identification and significance of coal-bed gas, San Juan Basin, northwestern New Mexico and southwestern Colorado, in Fassett, J.E., ed., Geology and coal-bed methane resources of the northern San Juan Basin, Colorado and New Mexico: Denver, Colorado, Rocky Mountain Association of Petroleum Geologists, p. 55-59.

Stach, E., Mackowsky, M. Th., Teichmuller, M., Taylor, G.H., Chandra, D., and Teichmuller, R., 1982, Stach's textbook of coal petrology, 3rd edition: Berlin-stuttgart, Borntraeger, $535 \mathrm{p}$.

Thaden, R.E., and Zech, R.S., 1984, Preliminary structure contour map on the base of the Cretaceous Dakota sandstone in the San Juan Basin and vicinity, New Mexico, Arizona, Colorado, and Utah: U.S. Geological Survey Miscellaneous Field Studies Map MF-1673.

Whyte, M.R., and shomaker, J.W., 1977, A geological appraisal of the deep coals of the Menefee Formation of the San Juan Basin, New Mexico, in Fassett, J.E., ed., supplement to san Juan Basin III: New Mexico Geological Society 28th Field Conference Guidebook, p. 41-48. 
Vitrinite reflectance values for coal from drill cuttings, san Juan Basin, New Mexico; depths reported in feet; $U$. = upper part, L. = lower part, Hog. Mt. Tongue = "Hogback Mountain Tongue" of the Menefee Formation

T. 23 N., R. 1 W., Sec. 15

Mobil Oil Corporation Badland Hills 15 No. 1

\begin{tabular}{|c|c|c|c|}
\hline$\frac{\text { Sampled Interval (s) }}{\text { U. Menefee } 4920-30}$ & $\frac{\% \mathrm{Rm}}{0.60}$ & $\begin{array}{l}\text { No. of } \\
\text { readings } \\
41\end{array}$ & $\begin{array}{l}\text { Standard } \\
\text { deviation } \\
0.06\end{array}$ \\
\hline L. Menefee 5290-5300 & 0.57 & 43 & 0.03 \\
\hline \multicolumn{4}{|c|}{$\begin{array}{l}\text { T. } 23 \text { N., R. } 7 \text { W. , Sec. } 15 \\
\text { BCo, Inc. Betty B' No. } 2\end{array}$} \\
\hline$\frac{\text { Sampled Interval(s) }}{\text { Fruitland } 1880-1920}$ & $\frac{\% \mathrm{Rm}}{0.44}$ & $\begin{array}{l}\text { No. of } \\
\text { readings } \\
31\end{array}$ & $\begin{array}{l}\text { Standard } \\
\text { deviation } \\
0.03\end{array}$ \\
\hline Menefee $3940-50$ & 0.44 & 5 & 0.04 \\
\hline \multicolumn{4}{|c|}{$\begin{array}{l}\text { T. } 23 \text { N. , R. } 7 \text { W. Sec. } 21 \\
\text { BCo, Inc. Federal'B No. } 7\end{array}$} \\
\hline$\frac{\text { Sampled Interval (s) }}{\text { Fruitland } 2050-2110}$ & $\frac{\% \mathrm{Rm}}{0.47}$ & $\begin{array}{l}\text { No. of } \\
\text { readings } \\
35\end{array}$ & $\begin{array}{l}\text { Standard } \\
\text { deviation }\end{array}$ \\
\hline Menefee $3920-30$ & 0.50 & 37 & 0.03 \\
\hline
\end{tabular}

T. 23 N., R. 7 W. , Sec. 29

Yates Petroleum Co. Henry AGC Fed. No. 1

\begin{tabular}{|c|c|c|c|}
\hline$\frac{\text { Sampled Interval (s) }}{\text { Fruitland 1528-1534 }}$ & $\frac{\% \mathrm{Rm}}{0.46}$ & $\begin{array}{l}\text { No. of } \\
\text { readings } \\
46\end{array}$ & $\begin{array}{l}\text { Standard } \\
\text { deviation } \\
0.03\end{array}$ \\
\hline U. Menefee 3049-3054 & 0.57 & 41 & 0.03 \\
\hline L. Menefee 3800-3810 & 0.58 & 42 & 0.02 \\
\hline $\begin{array}{l}\text { T. } 23 \text { N. R. } 9 \text { W. } S E \\
\text { Yates Hurricane Fed. }\end{array}$ & & & \\
\hline$\frac{\text { Sampled Interval (s) }}{\text { Fruitland } 1080-1100}$ & $\frac{\% \mathrm{Rm}}{0.46}$ & $\begin{array}{l}\text { No. of } \\
\text { readings } \\
33\end{array}$ & $\begin{array}{l}\begin{array}{l}\text { standard } \\
\text { deviation }\end{array} \\
0.03\end{array}$ \\
\hline L. Menefee 3636-3640 & 0.51 & 34 & 0.03 \\
\hline
\end{tabular}


T. 23 N., R. 10 W., Sec. 6

Dugan Production Co. Calgary No. 5

\begin{tabular}{|c|c|c|c|}
\hline$\frac{\text { Sampled Interval (s) }}{\text { U. Menefee } 2060-2070}$ & $\frac{2 . \mathrm{Rm}}{0.55}$ & $\begin{array}{l}\text { No. of } \\
\frac{\text { readings }}{34}\end{array}$ & $\begin{array}{l}\begin{array}{l}\text { Standard } \\
\text { deviation }\end{array} \\
0.03\end{array}$ \\
\hline L. Menefee $3280-3290$ & 0.56 & 33 & 0.02 \\
\hline
\end{tabular}

\begin{tabular}{|c|c|c|c|}
\hline$\frac{\text { Sampled Interval (s) }}{\text { L. Menefee } 3200-3220}$ & $\frac{8 \mathrm{gRm}}{0.54}$ & $\begin{array}{l}\text { No. of } \\
\text { readings } \\
36\end{array}$ & $\begin{array}{l}\begin{array}{l}\text { standard } \\
\text { deviation }\end{array} \\
0.03\end{array}$ \\
\hline \multicolumn{4}{|c|}{$\begin{array}{l}\text { T. } 23 \text { N., R. } 10 \text { W. , Sec. } 6 \\
\text { Dugan Production Co. Champ No. } 5\end{array}$} \\
\hline$\frac{\text { Sampled Interval (s) }}{\text { Fruitland } 950-960}$ & $\frac{\frac{8 \mathrm{Rm}}{0.44}}{0.4}$ & $\begin{array}{l}\text { No. of } \\
\frac{\text { readings }}{31}\end{array}$ & $\begin{array}{l}\begin{array}{l}\text { Standard } \\
\text { deviation }\end{array} \\
0.04\end{array}$ \\
\hline Hog. Mt. Tongue 1880-1890 & 0.52 & 33 & 0.03 \\
\hline Hog. Mt. Tongue 1910-1920 & 0.49 & 33 & 0.03 \\
\hline L. Menefee $3170-3180$ & 0.54 & 37 & 0.02 \\
\hline L. Menefee $3210-3220$ & 0.54 & 35 & 0.03 \\
\hline
\end{tabular}

T. 24 N., R. 1 W., Sec. 28

Mobil Expl. and Producing Harrington Fed. 28 No. $1 Y$

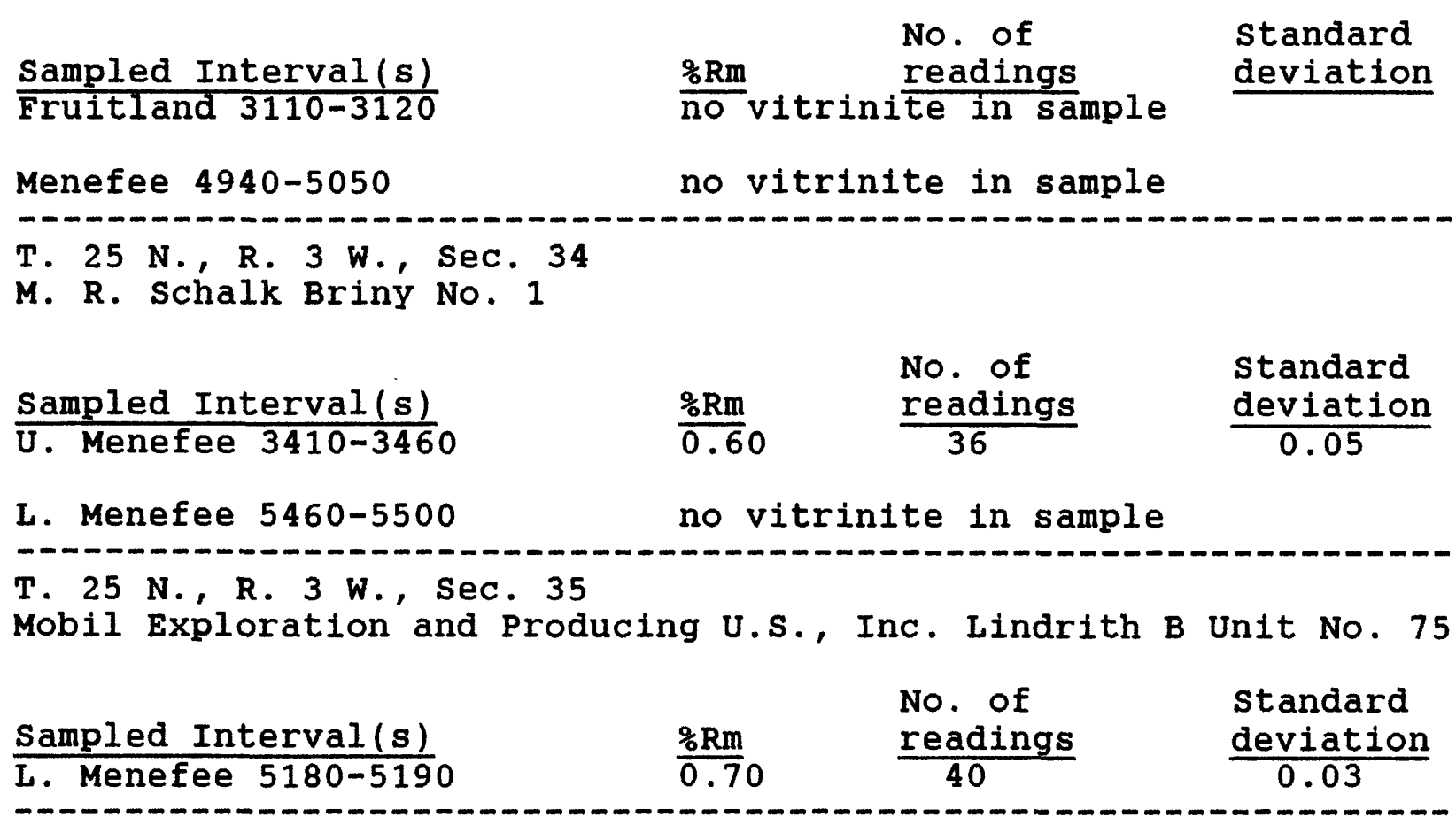


T. 25 N., R. 11 W., Sec. 32

Hixon Development Co. Jake Johnson No. 1

\begin{tabular}{|c|c|c|c|}
\hline$\frac{\text { Sampled Interval (s) }}{\text { Menefee } 3510-3540}$ & $\frac{\frac{9 R m}{0 R}}{0.39}$ & $\begin{array}{l}\text { No. of } \\
\text { readings } \\
30\end{array}$ & $\begin{array}{l}\text { standard } \\
\text { deviation } \\
0.04\end{array}$ \\
\hline $\begin{array}{l}\text { T. } 25 \mathrm{~N} . \text { R. } 11 \mathrm{~W} . \text { Sec. } 32 \\
\text { Hixon Development Co. Yomamas } \\
\text { Sampled Interval(s) } \\
\text { Fruitland } 1060-1090\end{array}$ & $\begin{array}{l}\text { No. } \\
\frac{8 \mathrm{Rm}}{0.46}\end{array}$ & $\begin{array}{l}\text { No. of } \\
\text { readings } \\
40\end{array}$ & $\begin{array}{l}\text { standard } \\
\text { deviation } \\
0.03\end{array}$ \\
\hline U. Hog. Mt. Tongue 2190-2220 & 0.46 & 32 & 0.02 \\
\hline L. Hog. Mt. Tongue 2530-2560 & 0.44 & 31 & 0.03 \\
\hline L. Menefee 3550-3580 & 0.49 & 50 & 0.06 \\
\hline
\end{tabular}

T. 26 N., R. 2 W., sec. 3

Amoco Production Co. Simmons Fed. Com. No. 1

$\begin{array}{llll}\text { Sampled Interval (s) } & \text { \%Rm } & \text { No. of } & \begin{array}{l}\text { Standrd } \\ \text { readings }\end{array}\end{array}$
L. Menefee 5629-?
0.80
35
0.04
T. 27 N., R. 6 W., Sec. 18
Unocal Rincon Unit No. 239

$\begin{array}{llll}\text { Sampled Interval }(\mathrm{s}) & \frac{\text { No. of }}{\text { Fruitland } 3090-3101} & \begin{array}{l}\text { Standard } \\ \text { readings }\end{array} & \frac{\text { deviation }}{45}\end{array}$

T. 27 N., R. 6 W., Sec. 21

Unocal Rincon Unit No. 240

\begin{tabular}{|c|c|c|c|}
\hline$\frac{\text { Sampled Interval (s) }}{\text { Fruitland } 3170-3190}$ & $\frac{\% \mathrm{Rm}}{0.64}$ & $\begin{array}{l}\text { No. of } \\
\text { readings } \\
43\end{array}$ & $\begin{array}{l}\text { standard } \\
\text { deviation } \\
0.04\end{array}$ \\
\hline
\end{tabular}

T. 27 N., R. 6 W., Sec. 22

Unocal Rincon Unit No. 241

\begin{tabular}{llll} 
Sampled Interval $(\mathrm{s})$ & $\frac{\% \mathrm{Rm}}{0.61}$ & $\frac{\text { No. of }}{\text { readings }}$ & $\begin{array}{l}\text { standard } \\
\text { deviation }\end{array}$ \\
\hline Fruitland $3072-3135$ & $\frac{0404}{0.04}$
\end{tabular}

T. 27 N., R. 6 W., sec. 23

Unocal Rincon Unit No. 242

Sampled Interval (s)

Fruitland 3205-3230

\begin{tabular}{lll}
$\frac{\% \mathrm{Rm}}{0.68}$ & $\begin{array}{l}\text { No. of } \\
\text { readings }\end{array}$ & $\begin{array}{l}\text { standard } \\
\text { deviation }\end{array}$ \\
\hline 0 & $\frac{03}{0.03}$ &
\end{tabular}


T. 27 N., R. 11 W., Sec. 16

Marathon oil Co. Schwerdtfeger No. 15C

\begin{tabular}{llll} 
Sampled Interval $(s)$ & $\begin{array}{l}\text { No. of } \\
\text { readings }\end{array}$ & $\begin{array}{l}\text { Standard } \\
\text { deviation }\end{array}$ \\
\hline Fruitland $1928-1938$ & $\frac{9 R m}{0.53}$ & $\frac{91}{0.03}$
\end{tabular}

T. 28 N., R. 5 W., Sec. 15

Meridian Oil, Inc. San Juan 28-5 Unit No. 201

$\begin{array}{lccc}\text { Sampled Interval }(\mathrm{s}) & \begin{array}{l}\text { No. of } \\ \text { readings }\end{array} & \begin{array}{c}\text { Standard } \\ \text { deviation }\end{array} \\ \text { Fruitland } 3517-3546 & \frac{8 \mathrm{Rm}}{0.77} & 41 & 0.06 \\ \text { Fruitland } 3620-3630 & 0.73 & 40 & 0.04 \\ \text { Fruitland } 3630-3640 & 0.72 & 40.04\end{array}$

T. 28 N., R. 5 W., Sec. 17

Meridian Oil, Inc. San Juan 28-5 Unit No. 203

\begin{tabular}{llll} 
Sampled Interval $(s)$ & $\frac{2}{2} \mathrm{Rm}$ & $\begin{array}{l}\text { No. of } \\
\text { readings }\end{array}$ & $\begin{array}{l}\text { Standard } \\
\text { deviation }\end{array}$ \\
\hline Fruitland $3420-3440$ & $\frac{0.75}{40}$ &
\end{tabular}

-

T. 28 N., R. 6 W., Sec. 19

Meridian Oil, Inc. San Juan 28-6 Unit No. 406

$\begin{array}{llll}\text { Sampled Interval (s) } & \begin{array}{l}\text { No. of } \\ \text { Fruitland } 3335-3339\end{array} & \begin{array}{l}\text { Standard } \\ \text { deadings }\end{array} & \frac{0.06}{41}\end{array}$

$---0.06$

T. 29 N., R. 7 W., Sec. 30

Meridian San Juan 29-7 Unit No. 513

\begin{tabular}{|c|c|c|c|}
\hline$\frac{\text { Sampled Interval (s) }}{\text { Fruitland } 2924-2930}$ & $\frac{8 \mathrm{Rm}}{0.70}$ & $\begin{array}{l}\text { No. of } \\
\frac{\text { readings }}{35}\end{array}$ & $\begin{array}{l}\begin{array}{l}\text { Standard } \\
\text { deviation }\end{array} \\
0.06\end{array}$ \\
\hline
\end{tabular}

T. 29 N., R. 8 W., Sec. 2

Meridian state Com. No. 100

\begin{tabular}{lccc} 
Sampled Interval (s) & $\begin{array}{l}\text { No. of } \\
\text { readings }\end{array}$ & $\begin{array}{l}\text { standard } \\
\text { deviation }\end{array}$ \\
\hline Fruitland $3001-3009$ & $\frac{2 R m}{0.72}$ & $\frac{41}{04}$ & -
\end{tabular}

T. 29 N., R. 9 W., sec. 21

D.J. Simmons Co. A.B. Geren No. 1

\begin{tabular}{lcc} 
Sampled Interval(s) & $\begin{array}{l}\text { No. of } \\
\text { readings }\end{array}$ & $\begin{array}{l}\text { Standard } \\
\text { deviation }\end{array}$ \\
\hline
\end{tabular}


T. 29 N., R. 9 W., Sec. 22

Meridian Oil, Inc. Grambling No. 720

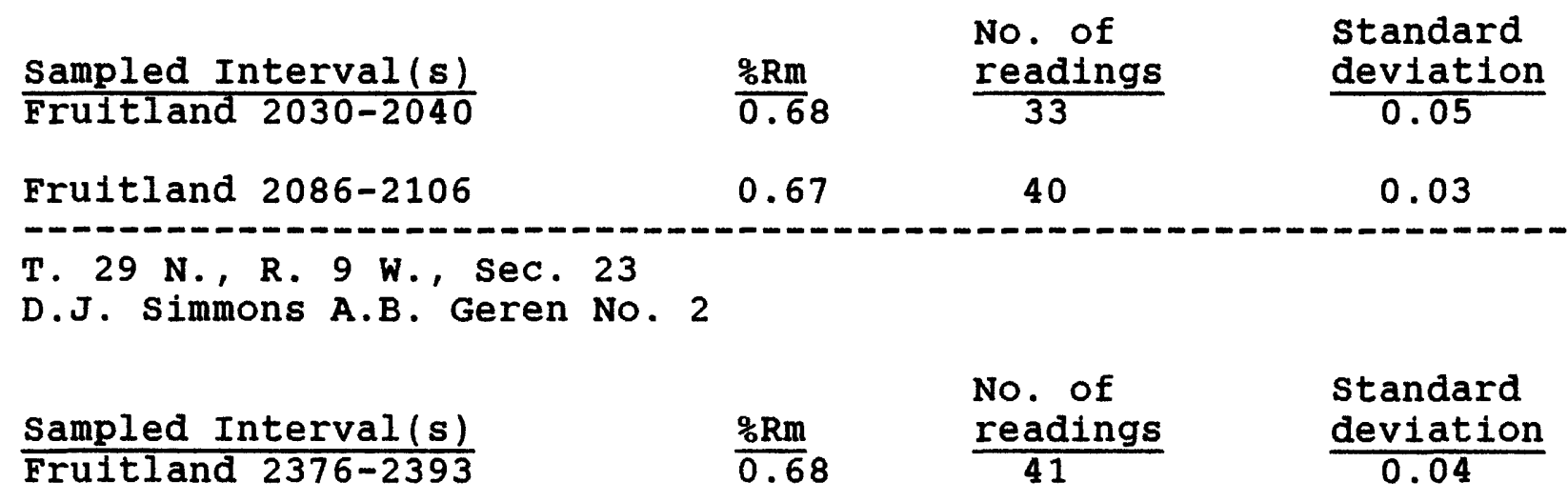

T. 29 N., R. 9 W., Sec. 29

D.J. Simmons Co. A.B. Geren No. 6

$\begin{array}{llll}\text { Sampled Interval (s) } & \frac{\% \mathrm{Rm}}{0.66} & \frac{\text { No. of }}{\text { readings }} & \begin{array}{l}\text { Standard } \\ \text { deviation }\end{array}\end{array}$

T. 29 N., R. 11 W., Sec. 27

Manana Marian $\mathbf{S}$ No. 1

\begin{tabular}{|c|c|c|c|}
\hline$\frac{\text { Sampled Interval (s) }}{\text { Fruitland } 1580-1585}$ & $\frac{\% \mathrm{Rm}}{0.59}$ & $\begin{array}{l}\text { No. of } \\
\text { readings } \\
41\end{array}$ & $\begin{array}{l}\text { Standard } \\
\text { deviation } \\
0.03\end{array}$ \\
\hline
\end{tabular}

T. 30 N., R. 6 W., Sec. 16

Meridian San Juan 30-6 Unit No. 407

\begin{tabular}{|c|c|c|c|}
\hline$\frac{\text { Sampled Interval (s) }}{\text { Fruitland } 3066-3092}$ & $\frac{8 \mathrm{Rm}}{0.97}$ & $\begin{array}{l}\text { No. of } \\
\text { readings } \\
41\end{array}$ & $\begin{array}{l}\text { standard } \\
\text { deviation } \\
\frac{0.04}{0.04}\end{array}$ \\
\hline
\end{tabular}

T. 30 N., R. 7 W., sec. 3

Blackwood \& Nichols Co. Ltd Northeast Blanco Unit No. 485

\begin{tabular}{|c|c|c|c|}
\hline$\frac{\text { Sampled Interval(s) }}{\text { Fruitland 2970-2975 }}$ & $\frac{\% \mathrm{Rm}}{0.88}$ & $\begin{array}{l}\text { No. of } \\
\text { readings } \\
40\end{array}$ & $\begin{array}{l}\text { Standard } \\
\text { deviation } \\
0.03\end{array}$ \\
\hline Fruitland 3075-3080 & 0.83 & 33 & 0.05 \\
\hline
\end{tabular}

T. 30 N., R. 7 W., sec. 29

Blackwood and Nichols Co., Ltd. Northeast Blanco Unit No. 477

\begin{tabular}{llll} 
Sampled Interval (s) & \%Rm & $\begin{array}{l}\text { No. of } \\
\text { readings }\end{array}$ & $\begin{array}{l}\text { standard } \\
\text { deviation }\end{array}$ \\
\hline Fruitland $3160-3165$ & $\frac{\text { oRm }}{0.76}$ & $\frac{04}{41}$ & $\frac{0.04}{}$
\end{tabular}


T. 30 N., R. 7 W., sec. 36

Meridian San Juan 30-6 Unit No. 468

$\begin{array}{llll}\text { Sampled Interval (s) } & \frac{8 \mathrm{Rm}}{0.91} & \frac{\begin{array}{l}\text { No. of } \\ \text { readings }\end{array}}{40} & \begin{array}{l}\text { Standard } \\ \text { deviation }\end{array} \\ \text { Fruitland } 3740-3744 & 0.04\end{array}$

T. 30 N., R. 8 W., Sec. 4

Amoco Production Co. Moore A No. 8

$\begin{array}{llcc}\text { Sampled Interval (s) } & \frac{8 \mathrm{Rm}}{0.76} & \frac{\begin{array}{l}\text { No. of } \\ \text { readings }\end{array}}{41} & \begin{array}{l}\text { Standard } \\ \text { deviation }\end{array} \\ \text { Fruitland 2927-2937 } & 0.82 & 40 & 0.03 \\ \text { Fruitland 3085-3106 } & 0.82 & \end{array}$

T. 30 N., R. 8 W., Sec. 5

Amoco Production Co. Moore B No. 3

\begin{tabular}{llll} 
Sampled Interval (s) & $\begin{array}{l}\text { No. of } \\
\text { readings }\end{array}$ & $\begin{array}{l}\text { standard } \\
\text { deviation }\end{array}$ \\
\hline Fruitiand 2999-3008 & $\frac{\% \mathrm{Rm}}{0.81}$ & $\frac{0.03}{35}$ &
\end{tabular}

T. 30 N., R. 8 W., Sec. 8

Amoco Production Co. Moore C No. 3

$\begin{array}{llcc}\text { Sampled Interval (s) } & \frac{\% \mathrm{Rm}}{\text { So. of }} & \begin{array}{l}\text { No. } \\ \text { readings }\end{array} & \begin{array}{l}\text { Standard } \\ \text { deviation }\end{array} \\ \text { Fruitland 2942-2948 } & \frac{0.65}{31} & 35 & 0.05\end{array}$

T. 30 N., R. 8 W., Sec. 12

Meridian Northeast Blanco Unit No. 437

\begin{tabular}{llll} 
Sampled Interval $(\mathbf{s})$ & $\frac{8 \mathrm{Rm}}{0.81}$ & $\frac{\begin{array}{l}\text { No. of } \\
\text { readings }\end{array}}{36}$ & $\begin{array}{l}\text { Standard } \\
\text { deviation }\end{array}$ \\
\hline Fruitland $2980-2995$ & 0.03
\end{tabular}

T. 30 N., R. 8 W., Sec. 25

Amoco Production Co. Florance $K$ No. 3

\begin{tabular}{llll} 
Sampled Interval (s) & $\begin{array}{l}\text { \%Rm } \\
\text { Fruitland 2907-2920 }\end{array}$ & $\begin{array}{l}\text { of } \\
\text { readings }\end{array}$ & $\begin{array}{l}\text { Standard } \\
\text { deviation }\end{array}$ \\
\hline 5 & 0.75 & 05
\end{tabular}

T. 30 N., R. 8 W., sec. 28

Amoco Production Company Gartner A No. 14 
T. 30 N., R. 8 W., Sec. 32

Meridian Beaver Lodge Com. No. 320

$\begin{array}{llll}\text { Sampled Interval(s) } & \text { No. of } & \begin{array}{l}\text { standard } \\ \text { deviation }\end{array} \\ \text { Fruitland 2390-2400 } & \frac{\text { \%Rm }}{0.69} & \frac{\text { readings }}{40} & \frac{0.05}{}\end{array}$

T. 30 N., R. 9 W., Sec. 1

Amoco Production Co. Pritchard A No. 10

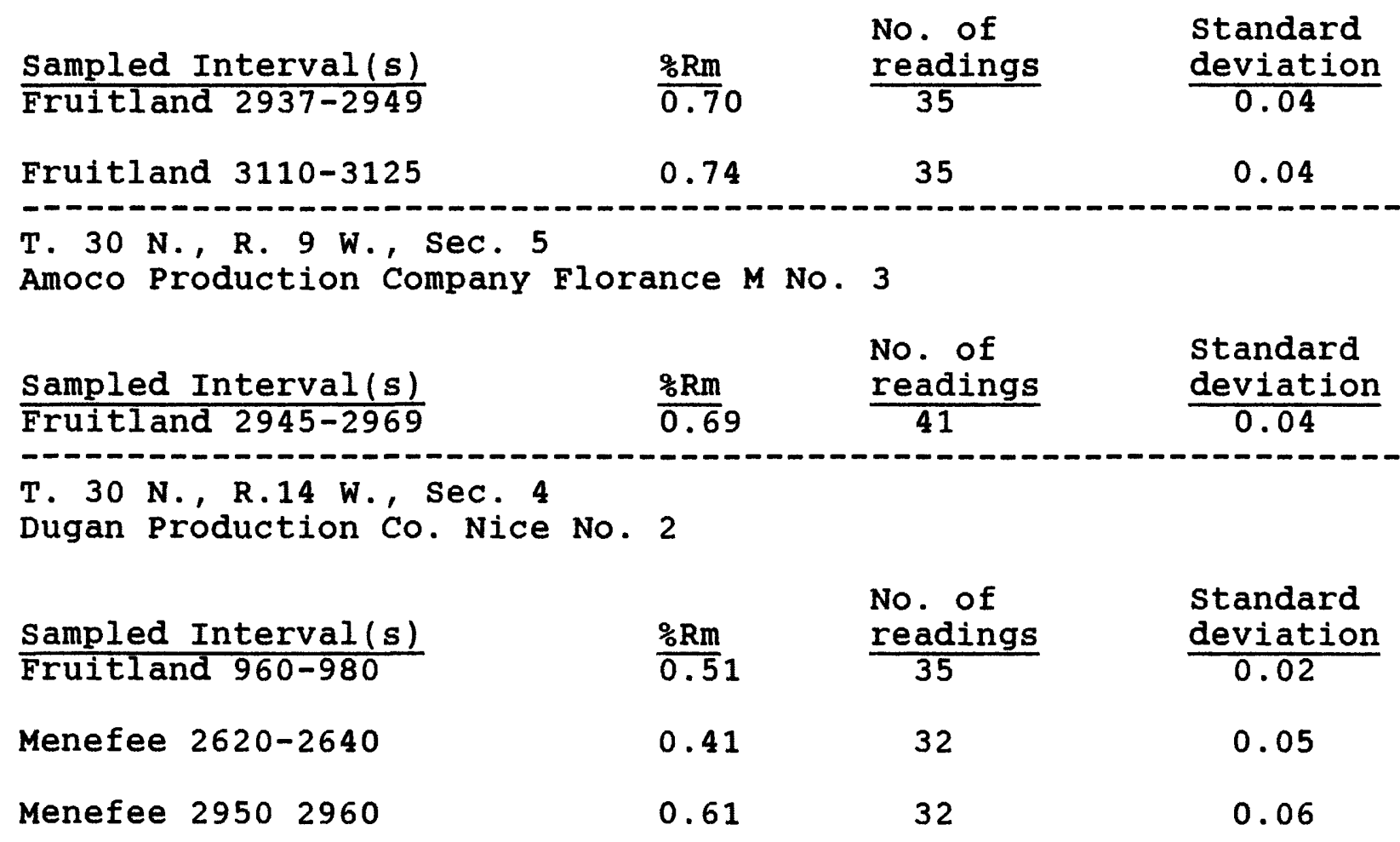

T. 30 N., R. 14 W., Sec. 11

Dugan Production Co. Blazer No. 2

\begin{tabular}{llll} 
Sampled Interval $(\mathrm{s})$ & $\begin{array}{l}\text { No. of } \\
\text { readings }\end{array}$ & $\begin{array}{l}\text { Standard } \\
\text { deviation }\end{array}$ \\
\hline Fruitland $1640-1680$ & $\frac{\% \mathrm{Rm}}{0.52}$ & $\frac{04}{32}$ & $\frac{04}{9}$
\end{tabular}

T. 31 N., R. 8 W., sec. 19

Union Texas Petroleum Quinn A No. 2

\begin{tabular}{llll} 
Sampled Interval(s) & $\%$ Rm & $\begin{array}{l}\text { No. of } \\
\text { readings }\end{array}$ & $\begin{array}{l}\text { Standard } \\
\text { deviation }\end{array}$ \\
\hline Fruitland $3140-3150$ & $\frac{0.77}{4}$ & $\frac{05}{4}$
\end{tabular}

T. 31 N., R. 8 W., Sec. 28

Amoco Production Co. Kernaghan B No. 5

\begin{tabular}{|c|c|c|c|}
\hline$\frac{\text { Sampled Interval (s) }}{\text { Fruitland } 3046-3056}$ & $\frac{\% \mathrm{Rm}}{0.82}$ & $\begin{array}{l}\text { No. of } \\
\frac{\text { readings }}{40}\end{array}$ & $\begin{array}{l}\text { standard } \\
\text { deviation } \\
0.04\end{array}$ \\
\hline Fruitland $3169-3184$ & 0.93 & 45 & 0.06 \\
\hline
\end{tabular}


T. 31 N., R. 8 W., sec. 30

Amoco Production Co. Kernaghan B No. 7

$\begin{array}{lccc}\text { Sampled Interval (s) } & \text { \%Rm } & \begin{array}{l}\text { No. of } \\ \text { readings }\end{array} & \begin{array}{l}\text { Standard } \\ \text { deviation }\end{array} \\ \text { Fruitland } 3216-3235 & 0.81 & 0.04 & 0.03 \\ \text { Fruitland } 3357-3367 & 0.83 & 35 & \end{array}$

T. 31 N., R. 8 W., sec. 33

Amoco Production Co. Kernaghan B No. 8

$\begin{array}{llcc}\text { Sampled Interval(s) } & \text { No. of } \\ \text { Fruitland } 3070-3078 & \frac{\% \mathrm{Rm}}{0.79} & \frac{1}{35} & \frac{\text { Standard }}{\text { deviation }} \\ \text { Fruitland } 3200-3224 & 0.80 & 45 & 0.05\end{array}$

T. 31 N., R. 9 W., sec. 27

Amoco Production Co. Pritchard B No. 5

\begin{tabular}{llll} 
Sampled Interval(s) & \%Rm & $\begin{array}{l}\text { No. of } \\
\text { readings }\end{array}$ & $\begin{array}{l}\text { standard } \\
\text { deviation }\end{array}$ \\
\hline Fruitland $3000-3013$ & $\frac{0.76}{40}$ & $\frac{0.05}{}$
\end{tabular}

T. 31 N., R. 9 W., Sec. 27

Amoco Production Co. Schwerdtfeger A No. 3

$\begin{array}{lccc}\text { Sampled Interval(s) } & \text { No. of } \\ \text { Fruitland 2723-2729 } & \frac{8 \mathrm{Rm}}{0.70} & \frac{\begin{array}{l}\text { Standard } \\ \text { deviation }\end{array}}{43} & \frac{0.06}{\text { Fruitland } 2870-2897} \\ \text { Fedings }\end{array}$

T. 31 N., R. 10 W., Sec. 21

Meridian Oil, Inc. Lambe Com. No. 200

$\begin{array}{llcc}\text { Sampled Interval(s) } & \text { No. of } \\ \text { Fruitland } 2720-2730 & \frac{\% \mathrm{Rm}}{0.66} & \frac{\text { standard }}{34} & \frac{0.04}{\text { deviation }} \\ \text { Fruitland 2900-2909 } & 0.71 & 40 & 0.04\end{array}$

T. 31 N., R. 10 W., sec. 23

Meridian Oil, Inc. Atlantic D Com B No. 205

$\begin{array}{lccc}\text { Sampled Interval(s) } & \text { No. of } \\ \text { Fruitland } 2980-2990 & \frac{\$ R m}{\text { readings }} & \begin{array}{l}\text { Standard } \\ \text { deviation }\end{array} \\ \text { Fruitland } 3130-3140 & 0.75 & 45 & 0.05 \\ - & & 0.03\end{array}$


T. 31 N., R. 11 W., Sec. 20

Meridian oil, Inc. Grenier No. 103

\begin{tabular}{|c|c|c|c|}
\hline$\frac{\text { Sampled Interval (s) }}{\text { Fruitland } 2290-2310}$ & $\frac{\% \mathrm{Rm}}{0.58}$ & $\begin{array}{l}\text { No. of } \\
\text { readings } \\
40\end{array}$ & $\begin{array}{l}\text { Standard } \\
\text { deviation } \\
0.04\end{array}$ \\
\hline Fruitland $2520-2540$ & 0.62 & 40 & 0.06 \\
\hline Fruitland $2540-2550$ & 0.66 & 40 & 0.06 \\
\hline
\end{tabular}

T. 31 N., R. 11 W., Sec. 36

Great Western Drilling Co. Pubco state Com. A No. 1

T. 32 N., R. 4 W., Sec. 15

Nassau Resources, Inc. Carracas Unit 15B No. 7

\begin{tabular}{llll} 
Sampled Interval (s) & No. of & $\begin{array}{l}\text { standard } \\
\text { deviation }\end{array}$ \\
\hline Fruitland $3351-3364$ & $\frac{\text { Readings }}{1.24}$ & $\frac{\text { reat }}{32}$ & $\frac{0.07}{}$
\end{tabular}

T. 32 N., R. 4 W., Sec. 16

Nassau Resources, Inc. Carracas Unit 16B No. 6

\begin{tabular}{|c|c|c|c|}
\hline Sampled Interval (s) & $\% \mathrm{Rm}$ & $\begin{array}{l}\text { No. of } \\
\text { readings }\end{array}$ & $\begin{array}{l}\text { standard } \\
\text { deviation }\end{array}$ \\
\hline
\end{tabular}

T. 32 N., R. 4 W., Sec. 17

Nassau Resources, Inc. Carracas Unit 17B No. 15

\begin{tabular}{llll} 
Sampled Interval $(\mathrm{s})$ & $\begin{array}{l}\text { No. of } \\
\text { readings }\end{array}$ & $\begin{array}{l}\text { Standard } \\
\text { deviation }\end{array}$ \\
\hline Fruitland $4180-4200$ & $\frac{\% \mathrm{Rm}}{1.30}$ & $\frac{05}{36}$ & $\frac{0}{0.05}$
\end{tabular}

T. 32 N., R. 4 W., Sec. 18

Nassau Resources, Inc. Carracas Unit 18B No. 15

\begin{tabular}{|c|c|c|c|}
\hline$\frac{\text { Sampled Interval (s) }}{\text { Fruitland } 4068}$ & $\frac{\% \mathrm{Rm}}{1.31}$ & $\begin{array}{l}\text { No. of } \\
\text { readings } \\
30\end{array}$ & $\begin{array}{l}\text { Standard } \\
\text { deviation } \\
0.08\end{array}$ \\
\hline Fruitland 4118-4128 & 1.27 & 30 & 0.06 \\
\hline
\end{tabular}

T. 32 N., R. 4 W., Sec. 18

Nassau Resources, Inc. Carracas Unit 18B No. 13

\begin{tabular}{llll} 
Sampled Interval $(\mathrm{s})$ & $\frac{\% \mathrm{Rm}}{1.14}$ & $\frac{\text { No. of }}{\text { readings }}$ & $\begin{array}{l}\text { Standard } \\
\text { deviation }\end{array}$ \\
\hline Fruitland 4160 & $\frac{05}{0.05}$
\end{tabular}


T. 32 N., R. 4 W., Sec. 19

Nassau Resources, Inc. Carracas Unit 19B No. 1

\begin{tabular}{|c|c|c|c|}
\hline$\frac{\text { Sampled Interval (s) }}{\text { Fruitland } 4095-4115}$ & $\frac{\frac{6}{\mathrm{Rm}}}{1.27}$ & $\begin{array}{l}\text { No. of } \\
\text { readings } \\
32\end{array}$ & $\begin{array}{l}\begin{array}{l}\text { Standard } \\
\text { deviation }\end{array} \\
0.04\end{array}$ \\
\hline
\end{tabular}

T. 32 N., R. 4 W., Sec. 20

Nassau Resources, Inc. Carracas Unit 20B No. 3

\begin{tabular}{llll} 
Sampled Interval $(s)$ & $\begin{array}{l}\text { No. of } \\
\text { readings }\end{array}$ & $\begin{array}{l}\text { Standard } \\
\text { deviation }\end{array}$ \\
\hline Fruitland $4134-4137$ & $\frac{8 \mathrm{Rm}}{1.28}$ & $\frac{07}{40}$ & $\frac{0}{2.07}$
\end{tabular}

T. 32 N., R. 4 W., Sec. 21

Nassau Resources, Inc. Carracas Unit 21B No. 5

\begin{tabular}{llll} 
Sampled Interval (s) & No. of & $\begin{array}{l}\text { Standard } \\
\text { deviation }\end{array}$ \\
\hline Fruitland $4055-4300$ & $\frac{\% R m}{1.27}$ & $\frac{\text { readings }}{35}$ & $\frac{0.05}{}$
\end{tabular}

T. 32 N., R. 4 W., Sec. 26

Nassau Resources, Inc. Carracas Unit 26B No. 16

\begin{tabular}{llll} 
Sampled Interval $(\mathrm{s})$ & $\frac{8 \mathrm{Rm}}{1.26}$ & $\begin{array}{l}\text { No. of } \\
\text { readings }\end{array}$ & $\begin{array}{l}\text { Standard } \\
\text { deviation }\end{array}$ \\
\hline Fruitland $3986-4003$ & $\frac{05}{1.05}$
\end{tabular}

-

T. 32 N., R. 4 W., Sec. 27

Nassau Resources, Inc. Carracas Unit 27B No. 5

\begin{tabular}{llll} 
Sampled Interval $(s)$ & $\frac{8}{6 \mathrm{Rm}}$ & $\begin{array}{l}\text { No. of } \\
\text { readings }\end{array}$ & $\begin{array}{l}\text { Standard } \\
\text { deviation }\end{array}$ \\
\hline Fruitland $4285 ?$ & 1.22 & $\frac{05}{33}$ &
\end{tabular}

T. 32 N., R. 4 W., Sec. 28

Nassau Resources, Inc. Carracas Unit 28B No. 5

$\begin{array}{llll}\text { Sampled Interval (s) } & \frac{\% \mathrm{Rm}}{1.30} & \frac{\begin{array}{l}\text { No. of } \\ \text { readings }\end{array}}{35} & \begin{array}{l}\begin{array}{l}\text { Standard } \\ \text { deviation }\end{array} \\ \text { Fruitland } 4045\end{array}\end{array}$

T. 32 N., R. 4 W., Sec. 30

Nassau Resources, Inc. Carracas Unit 30B No. 8

\begin{tabular}{lcc} 
Sampled Interval (s) & $\begin{array}{l}\text { No. of } \\
\text { readings }\end{array}$ & $\begin{array}{l}\text { Standard } \\
\text { deviation }\end{array}$ \\
\hline
\end{tabular}

T. 32 N., R. 4 W., sec. 32

Nassau Resources, Inc. Carracas Unit 32B No. 5

Sampled Interval(s)

Fruitland 3745-4125
No. of

$\frac{8 \mathrm{Rm}}{1.24} \quad \frac{\text { readings }}{40} \quad \frac{\text { deviation }}{0.06}$


T. 32 N., R. 4 พ., Sec. 34

Nassau Resources, Inc. Carracas Unit 34B No. 2

$\begin{array}{llll}\text { Sampled Interval }(\mathrm{s}) & \frac{q \mathrm{Rm}}{1.23} & \frac{\begin{array}{l}\text { No. of } \\ \text { readings }\end{array}}{40} & \begin{array}{l}\text { Standard } \\ \text { deviation }\end{array} \\ \text { Fruitland } 4160-4178 & 0.06\end{array}$

T. 32 N., R. 4 W., Sec. 35

Nassau Resources, Inc. Carracas Unit 35B No. 9

\begin{tabular}{llll} 
Sampled Interval (s) & $\frac{\% \mathrm{Rm}}{1.24}$ & $\begin{array}{l}\text { No. of } \\
\text { readings }\end{array}$ & $\begin{array}{l}\text { Standard } \\
\text { deviation }\end{array}$ \\
\hline Fruitland $3918-3934$ & $\frac{03}{1.03}$ &
\end{tabular}

T. 32 N., R. 4 w., Sec. 36

Nassau Resources, Inc. Carracas Unit 36B No. 15

\begin{tabular}{llll} 
Sampled Interval (s) & $\frac{\text { gRm }}{1.23}$ & $\frac{\begin{array}{l}\text { No. of } \\
\text { readings }\end{array}}{41}$ & $\begin{array}{l}\text { Standard } \\
\text { deviation }\end{array}$ \\
\hline Fruitland 3993-4006 & 0.06
\end{tabular}

T. 32 N., R. 5 w., Sec. 10

Nassau Resources, Inc. Carracas unit 10A No. 11

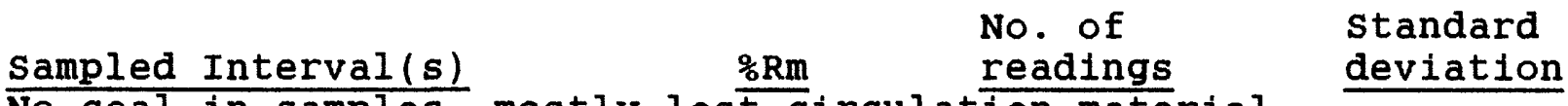

No coal in samples, mostly los t circulation material

T. 32 N., R. 5 W., Sec. 11

Nassau Resources, Inc. Carracas Unit 11A No. 9

\begin{tabular}{llll} 
Sampled Interval $(\mathrm{s})$ & $\frac{\% \mathrm{Rm}}{1.28}$ & $\frac{\begin{array}{l}\text { No. of } \\
\text { readings }\end{array}}{38}$ & $\begin{array}{l}\text { Standard } \\
\text { deviation }\end{array}$ \\
\hline Fruitland $4004-4255$ & 0.06
\end{tabular}

T. 32 N., R. 5 W., Sec. 13

Nassau Resources, Inc. Carracas Unit 13A No. 14

\begin{tabular}{llll} 
Sampled Interval $(\mathrm{s})$ & $\frac{\% \mathrm{Rm}}{\mathrm{F}}$ & $\begin{array}{l}\text { No. of } \\
\text { readings }\end{array}$ & $\begin{array}{l}\text { Standard } \\
\text { deviation }\end{array}$ \\
\hline Fruitland $4108-4128$ & $\frac{06}{1.24}$ &
\end{tabular}

T. 32 N., R. 5 W., Sec. 14

Nassau Resources, Inc. Carracas Unit 14A No. 1

\begin{tabular}{llll} 
Sampled Interval $(\mathrm{s})$ & $\frac{\% \mathrm{Rm}}{1.20}$ & $\frac{\begin{array}{l}\text { No. of } \\
\text { readings }\end{array}}{34}$ & $\begin{array}{l}\text { Standard } \\
\text { deviation }\end{array}$ \\
\hline Fruitland $3942-3966$ & 0.04
\end{tabular}

T. 32 N., R. 5 W., Sec. 14

Nassau Resources, Inc. Carracas Unit 14A No. 13

Sampled Interval (s)

Fruitland 3800-3824
No. of

$\frac{8 \mathrm{Rm}}{1.26} \quad \frac{\text { readings }}{30} \quad \frac{\text { deviation }}{0.05}$


T. 32 N., R. 5 W., Sec. 21

Nassau Resources, Inc. Carracas Unit 21A No. 15

\begin{tabular}{llll} 
Sampled Interval(s) & \%Rm & $\begin{array}{l}\text { No. of } \\
\text { readings }\end{array}$ & $\begin{array}{l}\text { Standard } \\
\text { deviation }\end{array}$ \\
\hline Fruitland $3690-3714$ & $\frac{1.17}{46}$ & $\frac{06}{0.06}$
\end{tabular}

T. 32 N., R. 5 W., Sec. 22

Nassau Resources, Inc. Carracas Unit 22A No. 16

\begin{tabular}{|c|c|c|c|}
\hline$\frac{\text { Sampled Interval (s) }}{\text { Fruitland } 3738-3781}$ & $\frac{\% \mathrm{Rm}}{1.18}$ & $\begin{array}{l}\text { No. of } \\
\text { readings } \\
41\end{array}$ & $\begin{array}{l}\text { standard } \\
\text { deviation } \\
\frac{0.07}{}\end{array}$ \\
\hline
\end{tabular}

T. 32 N., R. 5 W., Sec. 22

Nassau Resources, Inc. Carracas Unit 22A No. 13

\begin{tabular}{|c|c|c|c|}
\hline$\frac{\text { Sampled Interval (s) }}{\text { Fruitland } 3710-3730}$ & $\frac{\% \mathrm{Rm}}{1.18}$ & $\begin{array}{l}\text { No. of } \\
\text { readings } \\
40\end{array}$ & $\begin{array}{l}\text { standard } \\
\text { deviation } \\
0.05\end{array}$ \\
\hline
\end{tabular}

T. 32 N., R. 5 W. , sec. 23

Nassau Resources, Inc. Carracas Unit 23A No. 2

$\begin{array}{llll}\text { Sampled Interval }(\mathrm{s}) & \frac{\mathrm{Rm}}{1.08} & \frac{\text { No. of }}{\text { readings }} & \begin{array}{l}\text { Standard } \\ \text { deviation }\end{array}\end{array}$

T. 32 N., R. 5 W. , Sec. 23

Nassau Resources, Inc. Carracas Unit 23A No. 12

$\begin{array}{llll}\text { Sampled Interval (s) } & \begin{array}{l}\text { No. of } \\ \text { readings }\end{array} & \begin{array}{l}\text { Standard } \\ \text { deviation }\end{array} \\ \text { Fruitland } 3769-3940 & \frac{\% \mathrm{Rm}}{1.22} & \frac{04}{40} & \frac{0.04}{}\end{array}$

T. 32 N., R. 5 W. , sec. 26

Nassau Resources, Inc. Carracas Unit 26A No. 2

$\begin{array}{llll}\text { Sampled Interval(s) } & \text { \%Rm } & \begin{array}{l}\text { No. of } \\ \text { readings }\end{array} & \begin{array}{l}\text { Standard } \\ \text { deviation }\end{array} \\ \text { Fruitland } 3970 & \frac{1.21}{35} & \frac{06}{1.21}\end{array}$

T. 32 N., R. 5 W., sec. 27

Nassau Resources, Inc. Carracas Unit 27A No. 8

$\begin{array}{llll}\text { Sampled Interval }(s) & \text { No. of } & \text { standard } \\ \text { Fruitland } 3868-3882 & \frac{\text { Rm }}{1.18} & \frac{\text { readings }}{40} & \frac{\text { deviation }}{0.05}\end{array}$

T. 32 N., R. 5 W., sec. 35

Nassau Resources, Inc. Carracas Unit 35A No. 8

Sampled Interval (s)

Fruitland 3649-3673
No. of

$\frac{\% R m}{1.17} \quad \frac{\text { readings }}{36} \quad \frac{\text { deviation }}{0.04}$


T. 32 N., R. 6 W., Sec. 27

Meridian Oil, Inc. San Juan 32-5 Unit No. 108

\begin{tabular}{|c|c|c|c|}
\hline$\frac{\text { Sampled Interval (s) }}{\text { Fruitland } 3066-3080}$ & $\frac{\% \mathrm{Rm}}{1.15}$ & $\begin{array}{l}\text { No. of } \\
\text { readings } \\
40\end{array}$ & $\begin{array}{l}\begin{array}{l}\text { Standard } \\
\text { deviation }\end{array} \\
0.04\end{array}$ \\
\hline Fruitland $3150-3160$ & 1.22 & 40 & 0.06 \\
\hline Fruitland $3148-3158$ & 1.24 & 40 & 0.04 \\
\hline \multicolumn{4}{|c|}{$\begin{array}{l}\text { T. } 32 \text { N. R. } 11 \text { W. Sec. } 9 \\
\text { Union Texas Petroleum Ute No. } 39\end{array}$} \\
\hline$\frac{\text { Sampled Interval (s) }}{\text { Fruitland } 2500-2510}$ & $\frac{\% \mathrm{Rm}}{0.58}$ & $\begin{array}{l}\text { No. of } \\
\frac{\text { readings }}{41}\end{array}$ & $\begin{array}{l}\begin{array}{l}\text { Standard } \\
\text { deviation }\end{array} \\
0.04\end{array}$ \\
\hline Fruitland $2570-2590$ & 0.77 & 33 & 0.04 \\
\hline
\end{tabular}

T. 32 N., R. 11 W., sec. 12

Union Texas Petroleum Ute No. 34

$\begin{array}{llll}\text { Sampled Interval }(\mathrm{s}) & \frac{\% \mathrm{Rm}}{0.87} & \frac{\text { No. of }}{\text { readings }} & \begin{array}{l}\text { Standard } \\ \text { deviation }\end{array}\end{array}$

T. 32 N., R. 11 W., Sec. 12

Union Texas Petroleum Ute No. 33

Sampled Interval (s)

Fruitland 3060-3080

$\begin{array}{lll}\frac{8 R m}{0.84} & \begin{array}{l}\text { No. of } \\ \text { readings }\end{array} & \begin{array}{l}\text { Standard } \\ \text { deviation }\end{array} \\ 0.05 & \end{array}$

T. 32 N., R.11 W., sec. 24

Meridian Oil, Inc. Primo Mudge No. 100

$\begin{array}{lccc}\text { Sampled Interval }(\mathrm{s}) & \begin{array}{l}\text { No. of } \\ \text { readings }\end{array} & \begin{array}{c}\text { Standard } \\ \text { deviation }\end{array} \\ \text { Fruitland } 3070-3080 & \frac{\% \mathrm{Rm}}{0.76} & 0.04 & 0.03\end{array}$

T. 32 N., R. 12 W., Sec. 12

Great Western Drilling Co. J.E. Decker No. 9

Sampled Interval (s)

Fruitland 2629-2649

$\frac{8 R m}{0.75} \quad \begin{array}{ll}\text { No. of } \\ \text { readings }\end{array} \quad \begin{aligned} & \text { Standard } \\ & \text { deviation }\end{aligned}$

